\title{
Proceeding
}

Supplementary Issue: Spring Conferences of Sports Science. Costa Blanca Sports Science Events, 14-15 June 2019. Alicante, Spain.

\section{Jump and balance test in judo athletes with or without visual impairments}

\author{
ROCCO SPERA ${ }^{1}$, IMMACOLATA BELVISO ${ }^{1} \triangle$, FELICE SIRICO ${ }^{1}$, STEFANO PALERMI ${ }^{1}$, BRUNO \\ MASSA ${ }^{1}$, FILOMENA MAZZEO ${ }^{2}$, PIETRO MONTESANO ${ }^{3}$ \\ ${ }^{1}$ Department Public Health, Human Anatomy and Sport Medicine Division, University of Naples "Federico II", \\ Naples, Italy \\ ${ }^{2}$ Department of Science and Technology, University of Naples Parthenope, Naples, Italy \\ ${ }^{3}$ Department of Sport Sciences and Wellness, University of Naples Parthenope, Naples, Italy
}

\begin{abstract}
The study was conducted for four months with 8 judo athletes: 4 sighted people (4 M) and 4 visual impairment people ( $3 \mathrm{M}$ and $1 \mathrm{~F})$, aged between 18 and 52 (30.75 \pm 12.74$)$. According to the IBSA Visual Classification, all visual impairment subjects participating in our study were covered in the B1 category of visual deficit. This is a group represented by patients with no light perception in either eye up to light perception, and with an inability to recognize the shape of a hand at any distance or in any direction. From our cohort it was excluded subjects who have had low extremities musculoskeletal, neurological, or orthopaedic disorders in the previous six months. The aim of the study was to evaluate their balance with both closed and opened eyes and to set their lower limbs' strength: these are indispensable characteristics to carry out technical actions of judo. Anthropometric measures were compared between groups and data about jump protocol and balance protocol were analysed. Results of current research showed that postural stability is different in function of assessment with closed and open eyes. The result of the jump tests differs because the data do not show significant differences between long jump and high jump. The comparison between blinded and sighted judo athletes highlighted greater difficulties with eyes closed for sighted athletes than blinded ones. Keywords: Visual impairment people; Balance; Judo.
\end{abstract}

\section{Cite this article as:}

Spera, R., Belviso, I., Sirico, F., Palermi, S., Massa, B., Mazzeo, F., \& Montesano, P. (2019). Jump and balance test in judo athletes with or without visual impairments. Journal of Human Sport and Exercise, 14(4proc), S937S947. doi:https://doi.org/10.14198/ihse.2019.14.Proc4.56

Corresponding author. University of Naples "Federico II" - Department Public Health, Human Anatomy and Sport Medicine Division, via S. Pansini 5, second floor, 20th buildings, room 206, Naples, Italy.

E-mail: immacolata.belviso@unina.it

Supplementary Issue: Spring Conferences of Sports Science. Costa Blanca Sports Science Events, 14-15 June 2019. Alicante, Spain.

JOURNAL OF HUMAN SPORT \& EXERCISE ISSN 1988-5202

(c) Faculty of Education. University of Alicante

doi:10.14198/jhse.2019.14.Proc4.56 


\section{INTRODUCTION}

Disability status often does not allow to live a normal life and thus participating in physical activity programs could be very difficult. The possibility to take part into sport activities, regardless the impairments and disabilities, has a remarkable positive effect on quality of life (QoL) in subjects with disabilities. The first disabled people to take part in a sport competition were paralytics in 1948, and then in the following years the Paralympic games were organized. In general, the public is astonished by the performance of Paralympic athletes, given their extreme physical and technical capacities and despite of the presence of mild to severe physical disabilities (Loturco, Winckler, et al., 2015; Montesano et al., 2013).

Visual impairment people (VIP) experience a loss of function in activities of daily living (Brouwer, Sadlo, Winding et Hanneman, 2008), and visually impaired older people are more likely than sighted peers to move into residential settings, be physically dependent and have poor QoL (Ivers, Cumming, Mitchell et Attebo, 1998). The World Health Organization classifies visual impairment as a decrease in visual acuity or a reduction in visual field (World Health Organization, 2015). People with visual impairment may experience more handicap or disability, specifically related to mobility problems and reduced participation to the daily life activities (Owsley, McGwin, Lee, Wasserman et Searcey, 2009). Visual impairment in younger adults is linked to inactivity, higher body mass index (BMI), and poor balance reactions (Leissner, Coenen, Froehlich, Loyola et Cieza, 2014).

Blinded athletes can participate in sports divided into categories with reference to the condition of total blindness or a residual of the visual function.

One of the sports that allows to perform physical activity both for the total blinded people and for the visually impaired ones, divided into categories B1 and B2, is the judo: it is featured by balance, coordination and especially lower limbs strength. As happening in other sports (i.e., basketball (Montesano, Tafuri et Mazzeo, 2013), handball (Pietro, 2018), soccer (Montesano, 2016; Altavilla et al., 2018), volleyball), vertical jump performance represents an important index of explosive strength in lower limb muscles, a prerequisite for optimal performance during also judo competitions. Therefore, vertical jump tests are frequently used for the assessment of athletic performance, for the evaluation of training-related performance changes over time, and for talent identification (Maulder et Cronin, 2005). The gold standard for the assessment of vertical jump height is the application of force-plates. Compared to force-plates which are expensive and often immobile, inertial sensor systems such as the Gyko device are less expensive, mobile, and thus easy to administer in the field. Furthermore, this tool allows the assessment of jump performance on any surface (e.g., firm ground, sand or grass) and transfer facility with advantages to implement this assessment tool in the field (Lesinski, Muehlbauer et Granacher, 2016; Mazzeo et al., 2019).

Visual impairment people experience troubles in motor control and balance too: limited availability of visual information significantly impairs body balance, spatial orientation, and mobility, increasing the risk of dangerous falls and other causes of injuries (Maćkowiak, Osiński et Salamon, 2015). Sensory integration plays a vital role in the development of motor skills, physical fitness, and spatial orientation (Houglum, 2010). The somatosensory system, whose underlying mechanism is based upon the integration of information on reciprocal position of upper and lower limbs, is postulated to play a fundamental role in the process of compensating for visual impairment (Capicíková, Rocchi, Hlavacka, Chiari et Cappello, 2006). Information coming from proprioceptors, particularly those located within the lower limb postural muscles, are particularly valuable in the context of maintaining normal body posture. The receptors sensitive to vibration, touch, pressure, and stretching play a particularly important role for this aspect (Rauschecker, 1995). Balance 
control becomes a major problem for all blinded subjects but in older individuals with visual impairments this condition is even more severe (Lee et Scudds, 2003). Earlier studies reported that vision plays a major role in postural control (Choy, Brauer et Nitz, 2003) and that postural sway increases in the absence of vision (Hsu, Scholz, Schöner, Jeka et Kiemel, 2007) (Palm, Strobel, Achatz, von Luebken et Friemert, 2009). Postural stability is different if it is measured with eyes closed or opened, and the presence or absence of visual feedback also affects it.

There are numerous clinical tests to assess performance of the main types of balance (static postural control, dynamic- anticipatory, and reactionary- postural control). The single leg stance test (also referred as timed single limb stance, unipedal balance test, one leg stance test, and one-leg standing balance) is a simple test for measuring static aspects of balance that can be used in a variety of settings and requires minimal equipment or training (Springer, Marin, Cyhan, Roberts et Gill, 2007). Balance control is different in people who are partially or completely blinded (Ray, Horvat, Croce, Christopher Mason et Wolf, 2008), but balance training is effective and reduce postural sway in visually impaired individuals (Maćkowiak et al., 2015).

It is important to assess features of athletes with disabilities to help them in sports, allowing them to lead a normal life ( Mazzeo \& Volpe, 2016).

This research was conducted on eight athletes for a period of four months, four blind athletes (VIP group) (B1) and four sighted ones (Sighted Control), to verify the parameters of balance and strength of the lower limbs.

\section{METHODS AND MATERIALS}

\section{Participants}

The study included a group of 8 judo athletes: 4 sighted people (4 males) and 4 visual impairment people ( 3 males and 1 female), aged between 18 and 52 years $(30.75 \pm 12.74)$. According to the IBSA Visual Classification, all visual impairment subjects participating in our study represented a single category of visual deficit, B1: no light perception in either eye up to light perception, and an inability to recognize the shape of a hand at any distance or in any direction.

The athletes were recruited into a judo team, Naples NOIVED, participating in national competitions and international. In fact, the female athlete, aged 52 , is the national champion for her category.

Participants were excluded if they had any history of musculoskeletal, neurological, or orthopaedic disorder in the lower extremities within the preceding six months that might have affected their ability to execute the experimental protocol. Before the start of the study, written informed consent was obtained from the participants and their legal representatives. All measurements were conducted according to the latest version of the declaration of Helsinki

\section{Objectives}

The objectives of the study were to ascertain the ability to balance with the eyes closed and open and the strength of the lower limbs indispensable to carry out the technical actions of judo and win the races with the opponent's back on the tatami. 


\section{Training}

All the athletes trained in total three times a week, also taking care of the nutrition (23) and participated on average every sixty days in official competitions. Twice they did the training in the gym, for a total of about 140/150 minutes (70 minutes every single session), and once they had aerobic training like ergometer or treadmill. In the gym the physical and technical work was carried out in couples formed by a VIP and a sighted subject, that underwent the exact same kind of training.

\section{Measures}

Balance protocol

Prior to testing, all participants underwent a standardized five-minute warm-up consisting of submaximal plyometric and skipping exercises.

The balance protocol consists of Single Leg Stance (SLS) test, whose parameters were measured by Gyko (Microgate, Bolzano - Italy) and by a manual chronometer.

The SLS test was defined as standing on one foot without shoes with the contralateral knee bent and not touching the weight bearing leg. The investigator notes if the athlete's legs touched each other, the feet moved on the floor, the foot touches down, or the arms moved from their start position (Trojian et McKeag, 2006). They have to choose their dominant foot and they did not have to wear shoes. Two tests were performed: one with closed eyes, one with opened eyes. No rest time was given between the two tries. Parameters studied were ellipse area (EA) and length of centre of pressure (CoP) movement during monopodalic sway and time in seconds.

Jump protocol

Prior to testing, all participants underwent a standardized five minutes warm-up consisting of submaximal plyometric and skipping exercises.

The jump protocol consists of two kind of tests: single long jump and squat jump. All subjects were instructed to perform a long jump from a standing position (Almuzaini et Fleck, 2008). Standardized instructions were given to subjects that permitted them to begin the jump with bent knees and swing their arms to assist in the jump. A line drawn on a hard surface served as the starting line. The length of the jump was determined using a tape measure, which was affixed to the floor. Each subject was given 3 trials, and the distance of the best jump was measured, to the nearest $1 \mathrm{~cm}$, from the line to the point where the heel closest to the starting line landed. If the subject fell backward, the distance where the body part closest to the starting line touched the ground was measured as the jump's length. Each subject performed 3 jumps, whether a subject fell backward during an attempt.

In the squat jump, subjects executed a knee flexion until the thigh was parallel to the ground and, after an initial command, jumped as high as possible (Loturco, Nakamura, et al., 2015). 3 tries were performed, without rest between them; jump height was assessed using Gyko (Microgate, Bolzano - Italy).

\section{RESULTS}

Anthropometric parameters of the studied subjects are reported in Table 1. 
Table 1. Anthropometric and body mass parameters

\begin{tabular}{lcccc}
\hline & Age (years) & Height $(\mathbf{c m})$ & Weight $(\mathbf{k g})$ & BMl $(\mathbf{k g} / \mathbf{c m} 2)$ \\
\hline Sighted & $29.5( \pm 11.25)$ & $178.25( \pm 5.76)$ & $79( \pm 16.80)$ & $24.60( \pm 3.80)$ \\
VIP & $32( \pm 12.42)$ & $162.5( \pm 4.15)$ & $69.5( \pm 9.81)$ & $26.36( \pm 4.01)$ \\
p value & 0.6542 & 0.2149 & 0.7712 & 1.003 \\
\hline
\end{tabular}

Postural stability, with the ability to balance, is variable in function of measurements with closed and open eyes. The result of the jump tests is different because the data do not show significant differences between long jump and high jump (Table 2).

Table 2. Parameters balance and jump

\begin{tabular}{lccc}
\hline Variable & Sighted & VIP & P value \\
\hline EA eyes closed & $708.40( \pm 741.57)$ & $964.13( \pm 700.67)$ & 0.7728 \\
EA eyes opened & $186.15( \pm 144.52)$ & $913.61( \pm 577.08)$ & $0.0433^{*}$ \\
Length eyes closed & $148.24( \pm 99.52)$ & $263.85( \pm 156.71)$ & 0.2482 \\
Length eyes opened & $270( \pm 161.09)$ & $273.33( \pm 155.97)$ & 1.000 \\
Time eyes closed & $13.84( \pm 20.95)$ & $14.54( \pm 8.49)$ & 0.2482 \\
Time eyes opened & $89.77( \pm 38.68)$ & $11.25( \pm 6.53)$ & $0.020^{*}$ \\
Squat Jump & $33.74( \pm 7.16)$ & $27.34( \pm 7.79)$ & 0.3865 \\
Single Long Jump & $195.75( \pm 20.43)$ & $168.75( \pm 27.83)$ & 0.1489 \\
\hline
\end{tabular}

The comparison between blind and sighted judoki has revealed greater difficulties with eyes closed for sighted athletes than blind people (Figure 1).

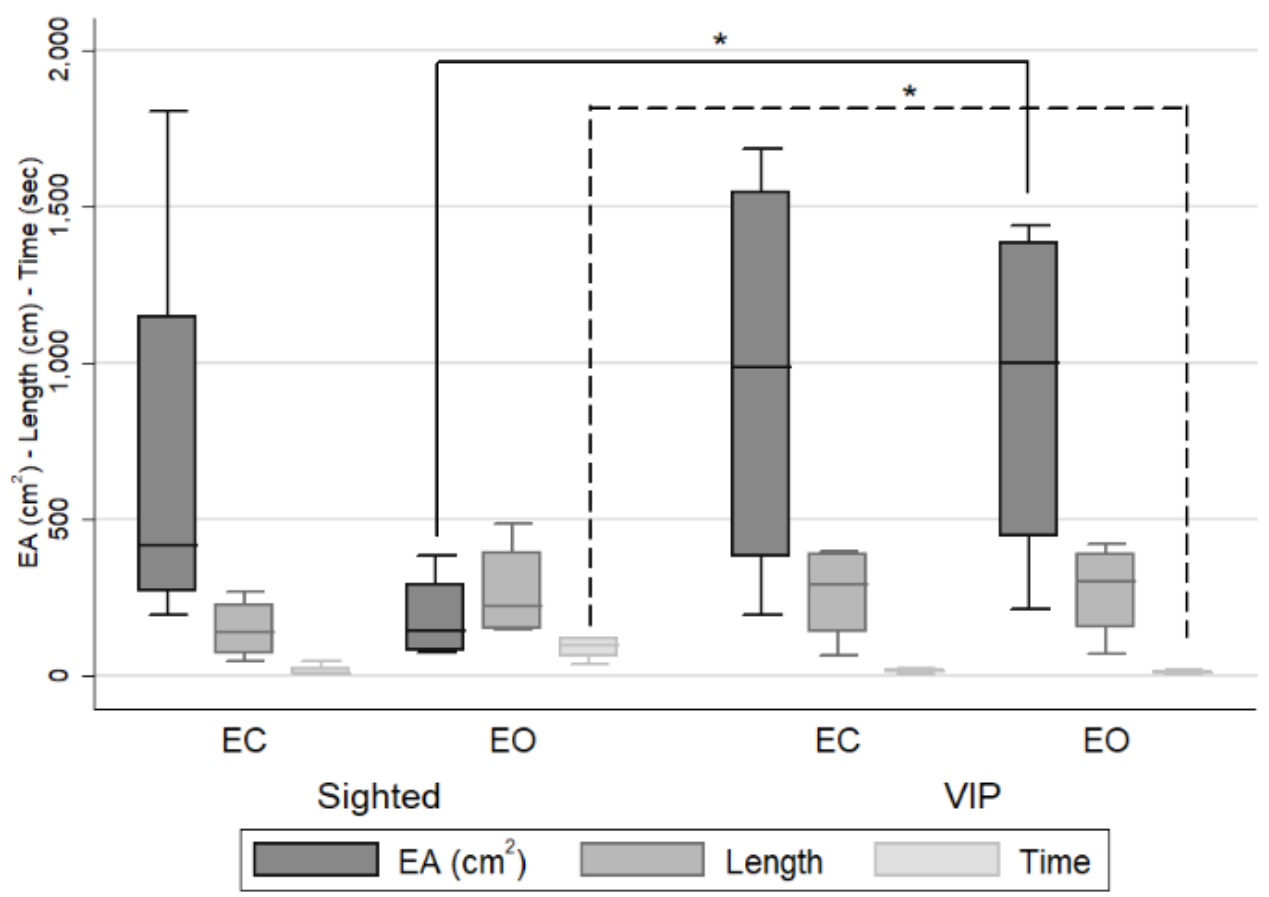

Figure 1. Balance protocol. 


\section{DISCUSSION}

Results of current research showed that postural stability is different in function of assessment with closed and open eyes. The result of the jump tests differs because the data do not show significant differences between long jump and high jump. The comparison between blinded and sighted judo athletes highlighted greater difficulties with eyes closed for sighted athletes than blinded ones.

The programs of sensorimotor training were revealed as an effective method of improving body balance and motor skills in humans (Maćkowiak et al., 2015). The results of previous studies suggest that demands placed upon the control systems during exercising on unstable ground improve both static and dynamic balance and consequently can reduce the risk of falling (Houglum, 2010; Mazzeo et al., 2018).

Numerous previous studies documented that blind individuals are characterized by a lower level of postural stability, manifested by increased area of CoP swaying in coronal and sagittal planes (Schmid, Nardone, De Nunzio, Schmid et Schieppati, 2007) (Marieb et Hoehn, 2004; Montesano \& Mazzeo, 2018). Vision has the strongest impact on the range of anterior-posterior sway, and the deprivation of visual information is reflected by marked disorders of balance (Maćkowiak et al., 2015). Sight is the quickest of our three main balance control systems to send information about the body's balance to the central nervous system, be it static or dynamic, and represents $70 \%$ of the body's sensory receptors (Sobry, Badin, Cernaianu, Agnani et Toussaint, 2014).

Palm et al. (Palm et al., 2009) showed that sighted people have a better postural stability when they keep their eyes opened. Because sighted people continuously use vision to maintain balance, they are generally considered as "visual addicts". The strategies used in the case of a sudden or gradual loss of sight remain unclear. We do not know either if the loss of sight will impact on the integrity of the balance system or if the body will generate enough compensation. However, we know that people who were born blinded benefit from a highly developed neuroplasticity of the (unused) visual cortex. In the case of visually impaired people (VIP), even though these subjects can move around and practice sports despite their handicap, we do not know if they are able to maintain an identical balance to that of sighted persons (Schwesig et al., 2011).

Balance training provides an efficient way to improve postural stability. In addition to the reduction in postural sway after several training sessions, balance training proved to be highly efficient in improving motor functions such as force production capacities (Sadowska, Stemplewski et Szeklicki, 2015).

The study conducted by Ray et al. (Ray et al., 2008) suggested that strongly VIP have a weaker postural stability and therefore modify their locomotion strategy. Schwesig et al. (Schwesig et al., 2011) found that their postural control is affected, but they found somatosensory and vestibular compensations for the absence of sight, especially among subjects with congenital blindness. For those subjects who were not born blinded, this compensation is not strong enough to ensure effective balance when compared to sighted people. In contrast, those blinded born subjects have an identical or even better balance than sighted persons with closed eyes (Schwesig et al., 2011). Both studies of Ray and Schwesig suggest some evidence about blind or virtually blind people but do not refer background regarding VIP.

However, when tested with vision control, the postural stability of sighted individuals turned out to be better than that of persons with visual impairment (Sobry et al., 2014). 
Decreased eyes opened time in SLS time is also associated with an increased risk for falls (Kurz, Lauber, Franke et Leukel, 2018) (Schwesig et al., 2011). Furthermore, the SLS conducted with eyes closed may yield other valuable information. Since patients with medical conditions that impair balance may rely heavily on vision to maintain their balance, it is possible that these patients would be at an increased risk for falls in conditions where visual input is inaccurate or eliminated, such as night-time ambulation (Springer et al., 2007). The SLS is described as a method of quantifying static balance ability (Schwesig et al., 2011). It is a valid measure (Sadowska et al., 2015). Even though the SLS appears to be used often in clinical settings to test static balance for a variety of reasons, limited normative data for the eyes open and closed conditions have been established with which to compare the tested values (Sadowska et al., 2015) (Vellas et al., s.d.).

A similar reflection must be made about the strength. Unlike the balance, blindness does not jeopardize jumping therefore, there is no difference between long jump and squat jump. There is, however, a correlation between the strength (Spearman coefficient 0.595): those who jump higher, jump even longer.

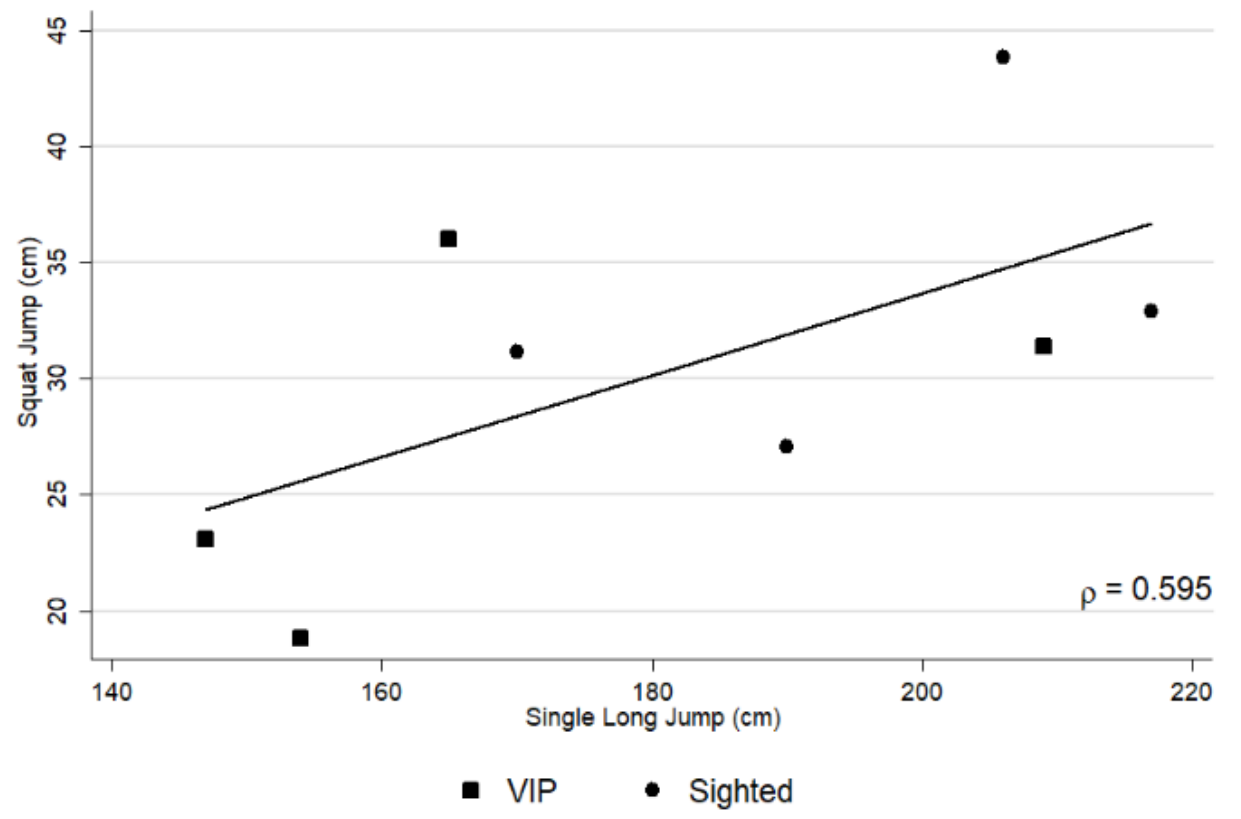

Figure 2. Jump protocol.

Muscle strength is essential for posture and stability (Ray et al., 2008). Muscle forces coupled with kinematic adjustments, especially around the knee and the ankle, are used to maintain stability and to prevent falls (Hurvitz, Richardson, Werner, Ruhl et Dixon, 2000). In fact, research showed that the best balance score occurred in individuals with stronger knee and ankle muscles (Newton, s.d.). Individuals with visual impairments have been described as consistently weaker than their sighted counterparts (Ray et al., 2008). They have more difficult to maintain stability if the command become higher. Additionally, as tasks difficult increase, different strategies are required to maintain posture (Horvat, Nocera, Ray et Croce, 2006). It has been suggested that muscle weakness in blinded individuals may be responsible for their diminished ability to quickly produce an adequate amount of force to compensate for movement variations or adjustments. It may be hypothesized that muscle weakness may force blinded individuals to perform different compensatory strategies to avoid placing their body in unstable positions. Indeed, blind individuals tend to use a hip strategy for balance control rather than the preferred ankle strategy (Ray et al., 2008). 
It is interesting to note that in our sample, although limited in number, 52 years-old women with visual impairment has been included. Her technical ability in Judo is high and she actually retained national title in her category. Nevertheless, it should be considered that our data demonstrated a markedly impact of blindness on balance impairment with reduced lower limb strength modification. This scenario could be useful in a public health point of view and trying to adopt specific training to improve balance in post-menopausal women should be encouraged. Although some pharmacological (lolascon, Sirico, Ferrante, Gimigliano et Gimigliano, 2010) and non-pharmacological treatment have demonstrated their ability to prevent fractures in this subset of population, we have to carefully assess also lifestyle and nutrition in these subsets of high-risk of falls women. Moreover, previous results demonstrate that subjects enrolled in individual sport, like judo, use less multivitamins than their active counterpart involved in team sports (Sirico et al., 2018) and these could determine deficiency in vitamin $\mathrm{D}$ or other vitamin and mineral supplementation, that requires to assess carefully the nutritional status too in these subjects with high-risk of fall.

\section{CONCLUSION}

The pilot study carried out on eight athletes, four blinded and four normal-sighted, who during the week carried out training in pairs, showed that, contrary to what one can imagine, postural stability, with the ability to balance, is variable in function of measurements with closed and open eyes. The result of the jump tests is different because the data do not show significant differences between long jump and high jump. The comparison between blind and sighted judoki has revealed greater difficulties with eyes closed for sighted athletes than blind people. This shows that vision loss significantly affects performance particularly if athletes are not blinded from birth but have progressively lost their sight. As regards the comparison, the actual difference in the jump test was determined by the subjective strength of the lower limbs. The final consideration concerns the quality of training and the proprioceptive stimulation oriented towards compensation through the other senses, the development of vicarious senses.

\section{ACKNOWLEDGMENTS}

The tests made comply with the current laws of the country in which they were performed.

\section{REFERENCES}

Altavilla, G., Mazzeo, F., D’Elia, F., \& Raiola, G. (2018). Physical commitment and specific work for each role in an elite soccer team. Journal of Physical Education and Sport, 18(2), 570-574. https://doi.org/10.7752/jpes.2018.02083

Almuzaini, K. S. et Fleck, S. J. (2008). Modification of the Standing Long Jump Test Enhances Ability to Predict Anaerobic Performance. Journal of Strength and Conditioning Research, 22(4), 1265-1272. https://doi.org/10.1519/jsc.0b013e3181739838

Brouwer, D. M., Sadlo, G., Winding, K. et Hanneman, M. I. G. (2008). Limitations in Mobility: Experiences of Visually Impaired Older People. British Journal of Occupational Therapy, 71(10), 414-421. https://doi.org/10.1177/030802260807101003

Capicíková, N., Rocchi, L., Hlavacka, F., Chiari, L. et Cappello, A. (2006). Human postural response to lower leg muscle vibration of different duration. Physiological research, 55 Suppl 1, S129-34. Retrieved at http://www.ncbi.nlm.nih.gov/pubmed/17177621

Choy, N. L., Brauer, S. et Nitz, J. (2003). Changes in postural stability in women aged 20 to 80 years. The journals of gerontology. Series A, Biological sciences and medical sciences, 58(6), 525-30. https://doi.org/10.1093/gerona/58.6.m525 
Horvat, M., Nocera, J., Ray, C. et Croce, R. (2006). Comparison of Isokinetic Peak Force and Power in Adults with Partial and Total Blindness. Perceptual and Motor Skills, 103(1), 231-237. https://doi.org/10.2466/pms.103.1.231-237

Houglum, P. A. (2010). Therapeutic exercise for musculoskeletal injuries. Human Kinetics.

Hsu, W.-L., Scholz, J. P., Schöner, G., Jeka, J. J. et Kiemel, T. (2007). Control and Estimation of Posture During Quiet Stance Depends on Multijoint Coordination. Journal of Neurophysiology, 97(4), 3024-3035. https://doi.org/10.1152/in.01142.2006

Hurvitz, E. A., Richardson, J. K., Werner, R. A., Ruhl, A. M. et Dixon, M. R. (2000). Unipedal stance testing as an indicator of fall risk among older outpatients. Archives of physical medicine and rehabilitation, 81(5), 587-91. https://doi.org/10.1016/s0003-9993(00)90039-x

Iolascon, G., Sirico, F., Ferrante, A., Gimigliano, R. et Gimigliano, F. (2010). Risedronate's efficacy: from randomized clinical trials to real clinical practice. Clinical cases in mineral and bone metabolism : the official journal of the Italian Society of Osteoporosis, Mineral Metabolism, and Skeletal Diseases, 7(1), 19-22. Retrieved at http://www.ncbi.nlm.nih.gov/pubmed/22461286

Ivers, R. Q., Cumming, R. G., Mitchell, P. et Attebo, K. (1998). Visual impairment and falls in older adults: the Blue Mountains Eye Study. Journal of the American Geriatrics Society, 46(1), 58-64. https://doi.org/10.1111/j.1532-5415.1998.tb01014.x

Kurz, A., Lauber, B., Franke, S. et Leukel, C. (2018). Balance Training Reduces Postural Sway and Improves Sport-specific Performance in Visually Impaired Cross-Country Skiers. Journal of Strength and Conditioning Research, 1. https://doi.org/10.1519//sc.0000000000002597

Lee, H. K. M. et Scudds, R. J. (2003). Comparison of balance in older people with and without visual impairment. Age and ageing, 32(6), 643-9. https://doi.org/10.1093/ageing/afg110

Leissner, J., Coenen, M., Froehlich, S., Loyola, D. et Cieza, A. (2014). What explains health in persons with visual impairment? Health and Quality of Life Outcomes, 12(1), 65. doi:10.1186/1477-7525-1265. https://doi.org/10.1186/1477-7525-12-65

Lesinski, M., Muehlbauer, T. et Granacher, U. (2016). Concurrent validity of the Gyko inertial sensor system for the assessment of vertical jump height in female sub-elite youth soccer players . BMC Sports Science, Medicine and Rehabilitation, 8(1), 35. https://doi.org/10.1186/s13102-016-0061-x

Loturco, I., Nakamura, F. Y., Tricoli, V., Kobal, R., Cal Abad, C. C., Kitamura, K., ... González-Badillo, J. J. (2015). Determining the Optimum Power Load in Jump Squat Using the Mean Propulsive Velocity. PloS one, 10(10), e0140102. https://doi.org/10.1371/journal.pone.0140102

Loturco, I., Winckler, C., Kobal, R., Cal Abad, C. C., Kitamura, K., Veríssimo, A. W., ... Nakamura, F. Y. (2015). Performance changes and relationship between vertical jump measures and actual sprint performance in elite sprinters with visual impairment throughout a Parapan American games training season. Frontiers in Physiology, 6, 323. https://doi.org/10.3389/fphys.2015.00323

Maćkowiak, Z., Osiński, W. et Salamon, A. (2015). The Effect of Sensorimotor Training on the Postural Stability of Visually Impaired Women Over 50 Years of Age. Journal of Women \& Aging, 27(1), 68-80. https://doi.org/10.1080/08952841.2014.928140

Marieb, E. N. et Hoehn, K. (2004). Anatomie et physiologie humaines 5e | Pearson ERPI. Retrieved at https://pearsonerpi.com/fr/collegial-universitaire/anatomie-physiologie/anatomie-et-physiologiehumaines-5e

Maulder, P. et Cronin, J. (2005). Horizontal and vertical jump assessment: reliability, symmetry, discriminative and predictive ability. Physical Therapy in Sport, 6(2), 74-82. https://doi.org/10.1016/i.ptsp.2005.01.001

Mazzeo, F., \& Volpe, R. A. (2016). From gene doping to athlete biological passport. Sport Science, 9(2), 97-103. 
Mazzeo, F., Santamaria, S., \& Montesano, P. (2019). Gender difference, nutritional supplements and drug use in sport to enhancing performance: An italian revision over the last decade. Sport Mont,17(1), 69-73. https://doi.org/10.26773/smi.190212

Mazzeo, F., Monda, V., Santamaria, S., Nigro, E., Valenzano, A., Villano, I., . . Messina, G. (2018). Antidoping program: An important factor in the promotion and protection of the integrity of sport and athlete's health. Journal of Sports Medicine and Physical Fitness, 58(7-8), 1135-1145. https://doi.org/10.23736/S0022-4707.17.07722-2

Montesano, P., Tafuri, D., Esposito, A., Gigante, F., Salzano, E., Viscido, G., \& Mazzeo, F. (2013). Conditional abilities in young special olympics athletes who practice unified football. Journal of Physical Education and Sport, 13(4), 504-510. https://doi.org/10.7752/jpes.2013.04079

Montesano, P., \& Mazzeo, F. (2018). Pilates improvement the individual basics of service and smash in volleyball. Sport Mont, 16(3), 25-30. https://doi.org/10.26773/smj.181005

Montesano, P. (2016). Goalkeeper in soccer: performance and explosive strenght. Journal of Physical Education and Sport @ (JPES), 16(1), 230-233. https://doi.org/10.7752/jpes.2016.01036

Montesano, P. (2018). Monitoring and upgrading of coordinative and conditional capacities of young athletes practicing handball. Journal of Physical Education and Sport, 18, 465-468. https://doi.org/10.7752/ipes.2018.s165

Montesano, P., Tafuri, D. et Mazzeo, F. (2013). Improvement of the motor performance difference in athletes of weelchair Basketball. Journal of Physical Education and Sport, 13(3), 362-370. https://doi.org/10.7752/jpes.2013.03058

Newton, R. (s.d.). Review of tests of standing balance abilities. Brain injury, 3(4), 335-43. Retrieved at http://www.ncbi.nlm.nih.gov/pubmed/2684312

Owsley, C., McGwin, G., Lee, P. P., Wasserman, N. et Searcey, K. (2009). Characteristics of Low-Vision Rehabilitation Services in the United States. Archives of Ophthalmology, 127(5), 681. https://doi.org/10.1001/archophthalmol.2009.55

Palm, H.-G., Strobel, J., Achatz, G., von Luebken, F. et Friemert, B. (2009). The role and interaction of visual and auditory afferents in postural stability. Gait \& Posture, 30(3), 328-333. https://doi.org/10.1016/j.gaitpost.2009.05.023

Rauschecker, J. P. (1995). Compensatory plasticity and sensory substitution in the cerebral cortex. Trends in neurosciences, 18(1), 36-43. https://doi.org/10.1016/0166-2236(95)93948-w

Ray, C. T., Horvat, M., Croce, R., Christopher Mason, R. et Wolf, S. L. (2008). The impact of vision loss on postural stability and balance strategies in individuals with profound vision loss. Gait \& Posture, 28(1), 58-61. https://doi.org/10.1016/i.gaitpost.2007.09.010

Sadowska, D., Stemplewski, R. et Szeklicki, R. (2015). The Effect of Physical Exercise on Postural Stability in Sighted Individuals and Those Who Are Visually Impaired: An Analysis Adjusted for Physical Activity and Body Mass Index. Journal of Applied Biomechanics, 31(5), 318-323. https://doi.org/10.1123/jab.2014-0228

Schmid, M., Nardone, A., De Nunzio, A. M., Schmid, M. et Schieppati, M. (2007). Equilibrium during static and dynamic tasks in blind subjects: no evidence of cross-modal plasticity. Brain, 130(8), 2097-2107. https://doi.org/10.1093/brain/awm157

Schwesig, R., Goldich, Y., Hahn, A., Müller, A., Kohen-Raz, R., Kluttig, A. et Morad, Y. (2011). Postural Control in Subjects with Visual Impairment. European Journal of Ophthalmology, 21(3), 303-309. https://doi.org/10.5301/ejo.2010.5504

Sirico, F., Miressi, S., Castaldo, C., Spera, R., Montagnani, S., Di Meglio, F. et Nurzynska, D. (2018). Habits and beliefs related to food supplements: Results of a survey among Italian students of different education fields and levels. PLOS ONE, 13(1), e0191424. https://doi.org/10.1371/journal.pone.0191424 
Sobry, V., Badin, P., Cernaianu, S., Agnani, O. et Toussaint, M. (2014). Do visually impaired people have a static balance as effective as sighted people? NeuroRehabilitation, 35(4), 851-61. https://doi.org/10.3233/NRE-141181

Springer, B. A., Marin, R., Cyhan, T., Roberts, H. et Gill, N. W. (2007). Normative values for the unipedal stance test with eyes open and closed. Journal of geriatric physical therapy (2001), 30(1), 8-15. https://doi.org/10.1519/00139143-200704000-00003

Trojian, T. H. et McKeag, D. B. (2006). Single leg balance test to identify risk of ankle sprains. British journal of sports medicine, 40(7), 610-3; discussion 613. https://doi.org/10.1136/bjsm.2005.024356

Vellas, B. J., Rubenstein, L. Z., Ousset, P. J., Faisant, C., Kostek, V., Nourhashemi, F., ... Albarede, J. L. (s.d.). One-leg standing balance and functional status in a population of 512 community-living elderly persons. Aging (Milan, Italy), 9(1-2), 95-8. https://doi.org/10.1007/bf03340133

World Health Organization. (2015). ICD-10 Version: $2015 . \quad$ Retrieved at https://icd.who.int/browse10/2015/en\#/H54

This work is licensed under a Attribution-NonCommercial-NoDerivatives 4.0 International (CC BY-NC-ND 4.0). 